\title{
Dislexias adquiridas como referência para a análise das dificuldades de aprendizagem da leitura
}

\section{Acquired dyslexia as a reference for the analysis of reading disorders}

\author{
Sandra Regina Kirchner Guimarães*
}

\begin{abstract}
RESUMO
Vários autores têm tentado explicar as dificuldades de aprendizagem da leitura comparando o padrão de leitura dos sujeitos que apresentam essa dificuldade com o dos portadores de dislexia adquirida dos tipos fonológica (dificuldade na coordenação letra-som) e de superfície (distúrbio no processo de reconhecimento da forma da palavra). O presente estudo teve por objetivo identificar se o procedimento de leitura preferencialmente utilizado pelas crianças com dificuldades de aprendizagem dessa habilidade é diferente do procedimento utilizado pelos leitores normais. Foram formados três grupos de sujeitos: 20 crianças de $3^{\mathrm{a}} \mathrm{e} 4^{\mathrm{a}}$ séries com dificuldades de aprendizagem da leitura (grupo 1); 20 crianças da $1^{a}$ série com o mesmo nível de leitura dos sujeitos do grupo 1 (grupo 2); e 20 crianças de $3^{\mathrm{a}}$ e $4^{\mathrm{a}}$ séries com a mesma idade cronológica dos sujeitos do grupo 1 (grupo 3). A pesquisa consistiu em uma tarefa de leitura e duas provas de controle. Os escores obtidos revelaram que todos os sujeitos apresentaram melhor desempenho na leitura das palavras de alta frequiência e pior desempenho na leitura de palavras inventadas. Esses resultados indicam uso preferencial de um procedimento lexical (não-analítico) por todos os sujeitos. Identificou-se também que o grupo 1 apresentou menor facilidade em utilizar a mediação fonológica. Contudo, os resultados não mostram diferenças entre os procedimentos de leitura utilizados pelos sujeitos com e sem dificuldades de aprendizagem. Portanto, acredita-se que a comparação entre dislexias adquiridas e dificuldades de aprendizagem da lei-
\end{abstract}

* Departamento de Teoria e Fundamentos da Educação, Universidade Federal do Paraná, Curitiba - PR. 
tura não é o quadro teórico adequado para explicar este tipo de problema. Palavras-chave: transtornos da aprendizagem, dislexia do desenvolvimento, dificuldades de aprendizagem da leitura.

\begin{abstract}
Several authors have tried to explain reading disorders by comparing them to acquired dyslexia of different types: phonological (letter-sound coordination disorder), and surface (a disorder in the process of recognizing the word form). In this study we aimed at finding out if the preferred reading process used by children with reading disorders is different from the process used by normal readers. Three subject groups were formed: 20 children in the $3^{\text {rd }}$ and $4^{\text {th }}$ grades with reading disorders (Group 1); 20 children in the $1^{\text {st }}$ grade at the same reading level as the subjects in Group 1 (Group 2); and 20 children in the $3^{\text {rd }}$ and $4^{\text {th }}$ grades of the same chronological age as the subjects in Group 1 (Group 3). The study was based on a reading task and two control tests. The scores achieved revealed that all subjects performed best when reading high-frequency words, and performed worst when reading made up words. Such results indicate that all subjects prefer to use a lexical (non-analytical) procedure. We also learned that Group 1 experienced more difficulty than the other groups to use phonological mediation. However, the results show that there is no difference between reading procedures used by subjects with or without learning disorders. Therefore, we believe that the method of comparing acquired dyslexia and reading disorders is not suitable to theoretically explain this kind of problem.

Key-words: learning disorders, development dyslexia, reading disorders.
\end{abstract}

\title{
Introdução
}

Este estudo se insere no âmbito das pesquisas que procuram comparar características dos sujeitos com dificuldades de aprendizagem da leitura e escrita (dislexia do desenvolvimento) com as características próprias dos sujeitos com dislexias adquiridas.

A leitura, embora aparentemente simples, é uma atividade bastante complexa que exige vários processos perceptivos e cognitivos, bem como um bom conhecimento da língua e da gramática. 
A fim de explicar a capacidade para a leitura, é preciso considerar suas partes constitutivas. Para isso, devem-se definir dois componentes gerais: o acesso ao léxico e a compreensão. $\mathrm{O}$ acesso ao léxico é o processo de "reconhecimento" de uma palavra (é o acesso à palavra na memória permanente, dado um input gráfico impresso). A compreensão, por sua vez, é o resultado de vários processos que operam sobre os textos.

Assim, modelos de reconhecimento de palavras por adultos têm sido propostos na tentativa de caracterizar alguns dos processos mentais que possibilitam ao leitor identificar, compreender e pronunciar as palavras escritas, facilitando o entendimento dos aspectos do reconhecimento de palavras que estão comprometidos nos indivíduos disléxicos (RACK; SNOWLING; OLSON, 1992).

Dentre os modelos de reconhecimento de palavras, selecionou-se, como exemplo, o modelo elaborado por ELlis (1995), em virtude de ter sido extraído de diversos outros, salientando as áreas de amplo consenso entre os pesquisadores. Por trás desse modelo, identifica-se a idéia de que o reconhecimento de palavras é resultado de uma atividade orquestrada, a qual envolve diversos subsistemas cognitivos (ou módulos) que operam, pelo menos em parte, de forma independente. Assim, uma vez que diferentes módulos cognitivos estão envolvidos no reconhecimento de uma palavra, o dano cerebral ou o desenvolvimento anormal pode produzir leitores nos quais alguns aspectos da leitura são normais, enquanto outros estão prejudicados.

O modelo de ELLIS, mostrado a seguir na forma de diagrama, distingue três diferentes rotas entre a palavra impressa e a fala.

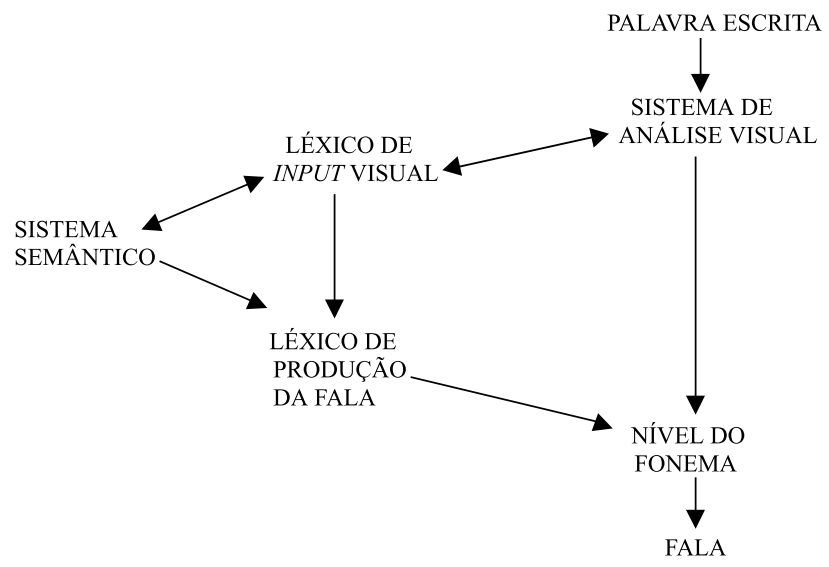

FIGURA 1 - MODELO DE RECONHECIMENTO DE PALAVRAS ESCRITAS ISOLADAS FONTE: ELLIS, A.W., 1995, p. 31. 
Para que se possa compreender a figura 1, é importante analisar as diferentes rotas que podem ser usadas para se ler uma palavra em voz alta.

Duas dessas rotas exigem que as palavras que estão sendo lidas sejam representadas no léxico de input visual e são utilizadas para converter as palavras familiares da escrita em som. A primeira rota, chamada lexical (ou semântica), é a leitura via significado, na qual a leitura é feita com a mediação da representação semântica. É utilizada a seguinte rota: palavra escrita $\rightarrow$ sistema de análise visual $\rightarrow$ léxico de input visual $\rightarrow$ sistema semântico $\rightarrow$ léxico de produção da fala $\rightarrow$ nível do fonema $\rightarrow$ fala.

A outra rota é a da leitura não-semântica, na qual a leitura é realizada a partir de uma conexão direta do léxico de input visual com o léxico de produção da fala. Portanto, é utilizada a seguinte rota: palavra escrita $\rightarrow$ sistema de análise visual $\rightarrow$ léxico de input visual $\rightarrow$ léxico de produção da fala $\rightarrow$ nível do fonema $\rightarrow$ fala.

Entretanto, se o item analisado visualmente não estiver representado no léxico de input visual, ele não será reconhecido como uma palavra e, conseqüentemente, só poderá ser lido pela rota sublexical (fonológica). Nessa rota (figura 1, à direita), a pronúncia é construída por meio da conversão dos segmentos ortográficos (i.e., grafemas simples, compostos ou dígrafos e seqüências de letras) em segmentos fonológicos. Depois da conversão, as unidades fonológicas são combinadas, formando um todo fonológico, e o acesso ao significado, caso ocorra, é obtido pela mediação da forma auditiva da palavra. Portanto, a rota fonológica é a seguinte: palavra escrita $\rightarrow$ sistema de análise visual $\rightarrow$ nível do fonema $\rightarrow$ fala.

\section{Fatores psicolingüísticos que afetam o reconhecimento de palavras}

Segundo ELLIS (1995), vários fatores determinam qual das rotas será usada para a leitura. Esses fatores são denominados "variáveis psicolingüísticas" (PARTZ, 1997, p. 59) e incluem, entre outros, a regularidade, a lexicalidade, a freqüência e o comprimento.

A regularidade diz respeito ao tipo de correspondência existente entre letra e som: relações biunívocas (palavras regulares) e relações cruzadas, as quais comportam as relações cruzadas previsíveis (palavras-regra) e as rela- 
ções cruzadas arbitrárias (palavras irregulares). Segundo o modelo de reconhecimento de palavras na leitura anteriormente exposto, palavras regulares podem ser lidas corretamente tanto pela rota sublexical (fonológica) quanto pela rota lexical. Entretanto, se a palavra que está sendo lida tem uma ortografia irregular, a rota sublexical tende a regularizá-la, produzindo uma pronúncia incorreta.

A lexicalidade apóia-se na distinção entre palavras reais e palavras inventadas (pseudopalavras), cujas estruturas ortográficas e fonológicas são admissíveis na língua portuguesa. No entanto, enquanto as palavras reais podem ser lidas tanto pela rota sublexical quanto pela rota lexical, as palavras inventadas não podem ser lidas pela rota lexical, pois, como não há representações ortográficas de palavras inventadas armazenadas no sistema lexical, elas devem ser lidas necessariamente pela rota sublexical.

A freqüência refere-se ao grau de ocorrência de certa palavra na língua. Como algumas palavras ocorrem com alta freqüência, enquanto outras ocorrem com baixa freqüência, as primeiras são mais familiares do que as segundas. Assim, quanto maior a freqüência de ocorrência das palavras, tanto maior a probabilidade de que elas sejam lidas pela rota lexical. Por outro lado, as palavras de baixa frequiência tendem a ser lidas pela rota sublexical, na medida em que não possuem representações ortográficas no léxico, pois essas representações são formadas a partir de exposições repetidas às palavras escritas.

O comprimento diz respeito ao tamanho do item (palavra ou pseudopalavra) e é definido, principalmente, em termos de número de letras ou de sílabas. A leitura pela rota fonológica é mais precisa para itens curtos que longos, enquanto a leitura pela rota lexical não sofre efeito do comprimento.

No próximo item, serão examinados os principais padrões de disfunção na leitura (dislexias adquiridas), pois a distinção entre eles tem sido feita em termos de danos aos componentes das diferentes rotas de leitura. 


\section{Principais padrões de disfunção na leitura: as dislexias adquiridas}

O termo dislexia adquirida é empregado para caracterizar um distúrbio de leitura encontrado em leitores adultos anteriormente capazes, causado por danos cerebrais, como por exemplo um acidente cérebro-vascular. De acordo com elLis (1995), os disléxicos possuem problemas nos módulos cognitivos utilizados na conversão da palavra escrita para o som e, dependendo do módulo afetado, têm-se diferentes padrões de disfunção na leitura.

Segundo PINHEIRo (1994) e MORAIS (1996), os principais padrões de disfunção na leitura podem ser divididos em dois grupos: dislexias periféricas e dislexias centrais.

As dislexias periféricas são transtornos nos quais os danos localizam-se no sistema de análise visual, provocando uma série de prejuízos na percepção das letras. Assim, os danos podem ocorrer no nível da identificação de letras (dislexia por negligência), no nível da análise visual (dislexia da atenção) ou no nível do processamento global e do reconhecimento de palavras como um todo (leitura letra-por-letra).

Já as dislexias centrais são transtornos nos quais, além do sistema de análise visual, partes de uma das rotas (fonológica ou lexical), ou mesmo das duas, está danificada. As dislexias em que apenas uma das rotas está prejudicada são denominadas síndromes de um único componente (PINHEIRO, 1994) por exemplo, a leitura não-semântica ou a dislexia fonológica. Quando ambas as rotas apresentam problemas, as dislexias são denominadas sindromes de componentes múltiplos - por exemplo, as dislexias de superfície e profunda.

A tabela 1 resume esses tipos de dislexia. 
TABELA 1 - AS DISLEXIAS ADQUIRIDAS

\begin{tabular}{|c|c|c|c|}
\hline \multicolumn{4}{|c|}{ (a) Dislexias Periféricas } \\
\hline Tipo de Dislexia & $\begin{array}{l}\text { Componentes do } \\
\text { modelo de leitura } \\
\text { afetados }\end{array}$ & Características & $\begin{array}{c}\text { Estímulos que causam } \\
\text { dificuldades }\end{array}$ \\
\hline $\begin{array}{l}\text { Dislexia por } \\
\text { negligência }\end{array}$ & Sistema & $\begin{array}{c}\text { Incapacidade de observar } \\
\text { a extremidade esquerda } \\
\text { das palavras }\end{array}$ & \\
\hline $\begin{array}{l}\text { Dislexia da } \\
\text { atenção }\end{array}$ & $\begin{array}{c}\text { de } \\
\text { Análise } \\
\text { Visual }\end{array}$ & $\begin{array}{l}\text { Dificuldade em focalizar } \\
\text { a atenção numa letra ou } \\
\text { palavra; as letras de uma } \\
\text { palavra podem juntar-se a } \\
\text { outra palavra }\end{array}$ & $\begin{array}{l}\text { Leitura de grupos de } \\
\text { palavras }\end{array}$ \\
\hline $\begin{array}{c}\text { Leitura } \\
\text { Letra-por-letra }\end{array}$ & & $\begin{array}{l}\text { Dificuldade em perceber a } \\
\text { forma global das palavras }\end{array}$ & $\begin{array}{c}\text { Palavras longas (reais e } \\
\text { inventadas) }\end{array}$ \\
\hline \multicolumn{4}{|c|}{ (b) Dislexias Centrais } \\
\hline Tipo de Dislexia & $\begin{array}{l}\text { Componentes do } \\
\text { modelo de leitura } \\
\text { afetados }\end{array}$ & Características & $\begin{array}{c}\text { Estímulos que causam } \\
\text { dificuldades }\end{array}$ \\
\hline $\begin{array}{c}\text { Leitura } \\
\text { não-semântica }\end{array}$ & Sistema semântico & $\begin{array}{c}\text { Falta de entendimentos } \\
\text { das palavras lidas }\end{array}$ & \\
\hline $\begin{array}{l}\text { Dislexia } \\
\text { fonológica }\end{array}$ & $\begin{array}{l}\text { Partes da rota } \\
\text { sublexical (conexão } \\
\text { entre o sistema de } \\
\text { análise visual e o } \\
\text { nível do fonema) }\end{array}$ & $\begin{array}{l}\text { Dificuldade na conversão } \\
\text { letra-som e erros de } \\
\text { "lexicalização" }\end{array}$ & $\begin{array}{c}\text { Palavras } \\
\text { não-familiares e palavras } \\
\text { inventadas }\end{array}$ \\
\hline $\begin{array}{l}\text { Dislexia de } \\
\text { superfície }\end{array}$ & $\begin{array}{l}\text { Partes da rota } \\
\text { lexical e da rota } \\
\text { sublexical } \\
\text { (fonológica) }\end{array}$ & $\begin{array}{l}\text { Leitura pela rota } \\
\text { fonológica (menos } \\
\text { danificada) e erros de } \\
\text { regularização }\end{array}$ & Palavras irregulares \\
\hline $\begin{array}{l}\text { Dislexia } \\
\text { profunda }\end{array}$ & $\begin{array}{l}\text { Partes da rota } \\
\text { sublexical, da rota } \\
\text { visual direta e da } \\
\text { rota semântica }\end{array}$ & $\begin{array}{l}\text { Erros semânticos, erros } \\
\text { visuais simples, erros } \\
\text { visuais-semânticos, erros } \\
\text { de derivação e } \\
\text { substituições de funções } \\
\text { gramaticais }\end{array}$ & $\begin{array}{l}\text { Palavras não-familiares, } \\
\text { palavras inventadas e } \\
\text { especialmente as palavras } \\
\text { abstratas e as palavras } \\
\text { funcionais (artigos, } \\
\text { pronomes, conjunções e } \\
\text { advérbios) }\end{array}$ \\
\hline
\end{tabular}

Dentre os tipos citados de dislexia adquirida, apenas a dislexia fonológica e a dislexia de superfície serão descritas de forma mais detalhada, por apresentarem interesse teórico para o presente estudo, na medida em que vários 
autores (COLTHEART et al., 1983; TEMPLE; MARSHALL, 1983; CASTLES; COLTHEART, 1993) têm buscado explicar os padrões de leitura e escrita dos sujeitos com dificuldades de aprendizagem (denominada dislexia do desenvolvimento) usando como referência os sintomas das dislexias adquiridas.

\section{Dislexia fonológica}

Os disléxicos fonológicos apresentam grande dificuldade na leitura de palavras não-familiares e de palavras inventadas, em decorrência de uma perda substancial na capacidade de fazer uso do procedimento de leitura sublexical, que é representado pela conexão entre o sistema de análise visual e o nível do fonema. As palavras familiares, por seu turno, são identificadas com razoável sucesso (TEMPLE; MARSHALL, 1983) - na leitura destas palavras, os pacientes apresentam um índice de precisão entre 70\% e 90\%.

Os disléxicos fonológicos cometem erros de "lexicalização", ou seja, palavras inventadas são lidas como palavras conhecidas e visualmente semelhantes.

De acordo com BODER (1973), a dislexia fonológica representa cerca de $67 \%$ dos quadros disléxicos. Esse fato "corrobora a importância da rota fonológica para a leitura, confirmando a necessidade de desenvolver procedimentos de intervenção para prevenir e remediar dificuldades fonológicas." (CAPOVILLA, 1999, p. 17).

ELLIS (1995), por sua vez, considera a dislexia fonológica adquirida de grande interesse teórico, devido à semelhança entre os sintomas desta e os apresentados pelas crianças diagnosticadas como tendo dislexia do desenvolvimento. Entretanto, o mesmo autor reconhece que a comparação entre as dislexias adquiridas e as do desenvolvimento exige muita cautela.

\section{Dislexia de superfície}

O termo "dislexia de superfície" foi proposto por MARSHALL e NEWCOMBE (1973) para designar um distúrbio, de moderado a severo, no processo de reconhecimento da palavra pela rota lexical e uma dificuldade de menor intensidade na realização da leitura em voz alta, pela rota fonológica. Por esse motivo, a leitura dos sujeitos com dislexia superficial é feita basicamente pela rota fonológica, ou seja, por meio da conversão letra-som.

CASTLES e COLTHEART (1993) enfatizam que já foram feitos vários relatos de sujeitos que podem ler palavras regulares e palavras inventadas (pseudopalavras) em voz alta, mas têm dificuldades na leitura de palavras irregulares (BEHRMANN; BUB, 1992; COLTHEART et al., 1983). 
De acordo com BADDELEY, LOGIE e ELLIS (1988), os disléxicos de superfície não são afetados pela lexicalidade, por aspectos semânticos, nem por referências concretas e imagináveis das palavras, mas por características da representação grafêmica, isto é, pelo comprimento da palavra e pela regularidade da correspondência letra-som.

Conforme explica ELLIS (1995), a leitura dos disléxicos de superfície segue o seguinte procedimento: decomposição das palavras em letras ou grupos de letras $\rightarrow$ conversão das letras em fonemas $\rightarrow$ pronúncia da seqüência sonora resultante. Mesmo a leitura das palavras familiares segue esse procedimento, pois são tratadas como se fossem desconhecidas.

Esse tipo de procedimento possibilita uma leitura de palavras regulares com mais sucesso do que a leitura das irregulares; estas, por sua vez, são pronunciadas de acordo com as regras usuais de conversão grafema-fonema, razão pela qual os pacientes cometem erros de regularização (CASTLES; COLTHEART, 1993).

\section{Dislexia adquirida e dislexia do desenvolvimento}

Para COLTHEART (1987), a aprendizagem da leitura pelas crianças normais pressupõe a utilização progressivamente melhor dos procedimentos lexical e sublexical.

A partir desse entendimento, CASTLES e COLTHEART (1993) apontam vários estudos, dentre eles os de COLTHEART et al. (1983) e TEMPLE e MARSHALL (1983), que tentaram explicar a dislexia do desenvolvimento em termos de dificuldades na aquisição de uma das rotas de leitura. Nessa perspectiva, os padrões de leitura apresentados pelas crianças disléxicas seriam semelhantes aos dos adultos com dislexia adquirida.

Diversos estudiosos que trabalharam com crianças disléxicas chegaram à conclusão de que os disléxicos não são todos iguais. Uma importante abordagem explicativa para essas diferenças focaliza as integridades e deficiências nos vários processos de leitura dos disléxicos. Por exemplo, BODER (1973), uma das primeiras defensoras da existênca de subtipos de dislexia, propôs uma distinção entre o que chamou de dislexia disfonética e diseidética. Os disléxicos disfonéticos seriam aqueles do tipo "fonológico", enquanto que os diseidéticos seriam do tipo de "superfície", isto é, apresentariam problemas, respectivamente, nas vias sublexical e lexical. 
Em relação à dislexia fonológica, CASTLES e COLTHEART (1993) afirmam que semelhanças funcionais entre dislexia adquirida e do desenvolvimento foram descritas, pela primeira vez, por TEMPLE e MARSHALL (1983). Eles estudaram o caso de uma garota de 17 anos (H.M.), que possuía inteligência média mas idade de leitura de 10 anos e 11 meses e idade de escrita em torno de 10 anos. Ela podia ler bem palavras familiares (tanto regulares quanto irregulares) em voz alta; entretanto, era incapaz de ler corretamente qualquer palavra inventada longa ou mesmo palavras reais não-familiares longas. Freqüentemente, na leitura de palavras inventadas, cometia lexicalizações, sendo que a maioria dos erros eram paralexias visuais (mudança da posição de letras e sons).

TEMPLE e MARSHALl (1983) analisaram também o material escrito por H.M. e verificaram que predominavam os erros do tipo fonético.

A conclusão de TEMPLE e TARSHALL (1983) foi de que as características de H.M. eram compatíveis com os casos de dislexia fonológica adquirida e que, portanto, poderia ser considerado como um caso de dislexia fonológica do desenvolvimento.

CASTLES e COLTHEART (1993), por sua vez, relatam que o primeiro pesquisador que apontou a semelhança entre a dislexia do desenvolvimento e a dislexia de superfície adquirida foi Holmes ${ }^{1}$ (1973); dez anos mais tarde, seu trabalho foi sustentado e ampliado por COLTHEART et al. (1983) com a descrição do caso da garota C.D. Esta paciente tinha 17 anos e possuía um QI superior a 100, mas sua idade de leitura era de 10 anos, apresentando melhor desempenho na leitura em voz alta de palavras regulares em relação às irregulares e, freqüentemente, cometendo erros de regularização. Os autores que estudaram o caso atribuíram a performance da paciente a uma dificuldade no uso do procedimento lexical e sustentaram a aplicação do termo dislexia de superfície do desenvolvimento para descrever sua condição.

O número de estudos de casos desses dois tipos de dislexia do desenvolvimento está se tornando abundante. SEYMOUR ${ }^{2}$ (apud COLTHEART, 1987) descreve cinco casos de dislexia de superfície do desenvolvimento e oito casos de dislexia fonológica do desenvolvimento.

Por outro lado, BRYANT e IMPEY (1986), com o objetivo de analisar as semelhanças entre os sintomas da dislexia adquirida e do desenvolvimento,

${ }^{1}$ HOLMES, J. M. Dyslexia: a neurolinguistic study of traumatic and developmental disorders of reading, Edinburgh, 1973, Doctoral Thesis - University of Edinburgh.

${ }^{2}$ SEYMOUR, P. K. H. Cognitive analysis of dyslexia, London: Routledge and Kegan Paul, 1986. 
retomaram os estudos de TEMPLE e MARSHALL (1983) e COLTHEART et al. (1983) e tentaram identificar se havia alguma coisa de diferente no padrão de leitura daquelas duas pacientes. Para isso, aplicaram em 16 leitores normais (com idade de leitura de 10 anos) os mesmos testes que haviam sido utilizados nos estudos acima referidos e encontraram evidências de que todos os sintomas descritos nos dois casos de dislexia também eram encontrados nos leitores normais.

De acordo com esses resultados, BRYANT e IMPEY (1986) concluíram que os padrões de leitura relatados por COLTHEART et al. (1983) e TEMPLE e MARSHALL (1983) não fornecem uma explicação sobre as causas das dificuldades de leitura. Por outro lado, esse experimento possibilitou a identificação de diferenças qualitativas entre os leitores ao longo de um continuum entre leitura fonológica e leitura de palavra inteira. Segundo BRYANT e IMPEY (1986), todas as crianças usam uma combinação dessas duas estratégias de leitura, entretanto foi descoberta uma correlação negativa entre elas, o que sugere a ocorrência de especialização em uma estratégia em detrimento de outra. Não obstante, os próprios BRYANT e IMPEY (1986) enfatizam a necessidade de mais estudos sobre esse tópico.

Assim, uma forma produtiva de interpretar o padrão de leitura das crianças disléxicas é compará-lo com o das crianças que não apresentam dificuldades na aprendizagem da leitura. Nessa perspectiva, a dislexia do desenvolvimento pode ser entendida como um atraso ou uma interrupção no processo de aquisição da leitura e da escrita. Por exemplo, FRITH (1984) hipotetizou que a dislexia, na sua forma clássica, representa uma retenção do desenvolvimento na fase logográfica, ou seja, a criança disléxica fracassa na fase alfabética em virtude dos seus déficits fonológicos.

Em suma, no contexto em que o presente estudo se insere não há consenso entre os pesquisadores quanto à adequação das comparações entre a dislexia adquirida e a do desenvolvimento. Por isso, a condução desta pesquisa teve como objetivo fornecer elementos de resposta às seguintes questões:

- Que procedimentos de leitura os sujeitos pesquisados utilizam preferencialmente: sublexical ou lexical?

- Os procedimentos adotados pelos sujeitos com dificuldades na aprendizagem da leitura são diferentes dos procedimentos adotados pelos demais sujeitos? 


\section{Método}

\section{Sujeitos}

O universo da pesquisa é formado por alunos da $1^{\mathrm{a}}, 3^{\mathrm{a}}$ e $4^{\mathrm{a}}$ séries do Ensino Fundamental de Escolas da Rede Municipal de Ensino de Curitiba que possuem salas de recursos ${ }^{3}$.

Do referido universo (que abrange 20 instituições escolares, agrupadas em sete núcleos), foram escolhidas aleatoriamente seis escolas. Após obter o consentimento dos responsáveis pelas instituições para a realização da investigação científica pretendida, a pesquisadora aplicou o Teste de Desempenho Escolar - TDE (sTEIN, 1994) nos alunos das salas de recursos, indicados pelos professores como tendo fraco desempenho em leitura. Com base nos resultados do TDE, o presente estudo ficou restrito a três escolas, onde foram identificados 20 alunos com dificuldades em leitura, os quais compuseram o grupo 1. Emparelhados a ele, foram selecionados outros dois grupos. Assim, foram formados três grupos, a saber:

- Grupo 1 - composto por 20 alunos (14 da $4^{\mathrm{a}}$ série e 6 da $3^{\mathrm{a}}$ série), com idade variando de 10 a 13 anos e 6 meses (média 11 anos e 6 meses), que freqüentavam salas de recursos e apresentavam dificuldades em leitura. Esses alunos, selecionados pelo TDE, obtiveram em leitura escores muito inferiores ao esperado para a sua série, embora tivessem obtido em aritmética um escore correspondente à série que freqüentavam.

- Grupo 2 - composto por 20 alunos da $1^{\text {a }}$ série com idade variando de 6 anos e 3 meses a 7 anos e 10 meses (média 7 anos e 1 mês), emparelhados com os sujeitos do grupo 1 pelo nível de leitura ${ }^{4}$. Também foram selecionados de acordo com os resultados do teste TDE,

${ }^{3}$ Em relação às salas de recursos, é importante ressaltar que elas "foram implantadas na Rede Municipal de Ensino a partir da necessidade de se oferecer atendimento aos alunos que apresentavam Dificuldades de Aprendizagem (...), cujas características não recomendavam atendimento em Classe Especial.” (CURITIBA, 1992, p. 50).

${ }^{4}$ Delineamentos de pesquisa com grupo de controle emparelhado pela idade de leitura têm sido usados por diversos pesquisadores (BRYANT; BRADLEY, 1985; 1987; BRYANT; NUNES; BINDMAN; 1997; GUIMARÃES, 2001; GUTHRIE,1973). Eles são apropriados para verificar se existem diferenças entre o padrão de leitura das crianças com dificuldades nessa área e o padrão de leitura dos leitores principiantes. 
tendo obtido escores em leitura adequados à sua série. Portanto, eram alunos que não apresentavam dificuldades de leitura e com idade cronológica inferior à dos alunos do grupo 1 .

- Grupo 3 - composto por 20 alunos (14 da $4^{a}$ série e 6 da $3^{a}$ série) provenientes das mesmas turmas (salas regulares) que os alunos do grupo 1 e com eles emparelhados de acordo com a idade cronológica, ou seja, com idade variando de 10 anos a 13 anos e 1 mês (média 11 anos e 1 mês). Para a seleção desses sujeitos, além do critério de idade, foi utilizado também o teste TDE, a fim de verificar se obtinham escores em leitura adequados às suas séries. Logo, eram alunos sem dificuldades em leitura e com a mesma idade cronológica dos sujeitos do grupo 1.

De acordo com os dados fornecidos pelas equipes pedagógico-administrativas das escolas, os alunos são oriundos de famílias com renda familiar de dois a cinco salários mínimos. Além disso, a escolaridade média de seus pais é a de $1^{\circ}$ grau. Com base nesses dados, considerou-se que não havia variação socioeconômica significativa entre os sujeitos pesquisados.

\section{Provas utilizadas}

O presente estudo foi baseado em dois tipos de provas experimentais:

\section{Medidas de controle ${ }^{5}$}

Foram usadas as mesmas medidas de controle utilizadas no estudo de REGO (1995): uma medida de inteligência, realizada por meio do teste das Matrizes Progressivas Coloridas de J. C. Raven, e uma tarefa de memória verbal. Entretanto, para os sujeitos com mais de 11 anos e 8 meses, foi utilizado o teste de Matrizes Progressivas - Escala Geral.

O objetivo de efetuar uma medida de controle da inteligência neste estudo deve-se ao fato de que a inteligência é uma variável freqüentemente associada ao sucesso escolar.

Já a tarefa de controle da memória verbal foi considerada em razão de a leitura envolver a retenção de informações verbais na memória a curto prazo. Sua

${ }^{5}$ Embora no delineamento do presente estudo não tenha sido previsto o controle da variação lingüística, ou seja, não foi avaliada a distância entre a fala da criança e a norma padrão utilizada na escola, acentua-se que esta é uma variável que pode estar significativamente relacionada ao desenvolvimento da leitura e da escrita (cf. BARRERA, 2000). Registra-se, portanto, a necessidade de incluir essa variável em futuros estudos. 
utilização objetivou controlar os eventuais efeitos desse tipo de memória. Para isso, foi solicitado às crianças que repetissem literalmente as 14 sentenças (duas de treinamento e doze de exame) ditas pela pesquisadora. Essas sentenças apresentavam tamanho e nível de complexidade crescentes. A primeira sentença que a criança deveria repetir era "Ontem choveu muito" e a última era "Carlos bebeu o leite porque ele gosta de brincar com Luiz quando está em casa".

Antes de iniciar a tarefa propriamente dita, a pesquisadora praticava com a criança as duas sentenças de treino. O escore de acerto podia variar de 0 a 12 , pois as crianças recebiam um ponto para cada uma das 12 sentenças que conseguissem repetir corretamente.

2. Tarefa de leitura de palavras reais e de palavras inventadas

Essa prova consiste na leitura, pelos sujeitos, de 36 palavras reais e 18 palavras inventadas apresentadas isoladamente em cartões individuais tamanho 7 x 4,5 cm, tendo sido as letras impressas em fonte Arial (18 pontos e caixa baixa).

As palavras que compõem a prova foram extraídas de PINHEIRO (1994), a qual, a partir de uma investigação de freqüência de ocorrência de palavras em livros de leitura da escola primária, dividiu as palavras em duas categorias (alta freqüência e baixa freqüência de ocorrência). Além disso, PINHEIRo (1994) dividiu as duas categorias acima em três subcategorias (regular, regra e irregular), de acordo com o relacionamento som-letra na ortografia da língua portuguesa. Destaca-se que essa última classificação leva em conta as irregularidades (relações não-biunívocas) na correspondência letra-som e som-letra que são marcadas na ortografia, pelo fato de que muitas das palavras classificadas como irregulares não causam nenhum problema na leitura ${ }^{6}$. De acordo com ALVARENGa (1988), a leitura, diferentemente da escrita, é na sua quase totalidade controlada por regras. Uma das exceções é que não existe regra que possibilite a previsão da leitura correta das palavras nas quais a unidade gráfica $x$ aparece em ambiente intervocálico. Portanto, ora $x$ representa /z/ como em exame, ora representa /š/ como em vexame, ora representa $/ \mathrm{ks} /$ como em boxe, ora não representa nenhum som específico, como em exceto.

As palavras inventadas que compõem a prova foram criadas a partir de alterações em uma ou duas letras das palavras reais de alta freqüência e foram

${ }^{6}$ É importante salientar que essa classificação foi mantida para repetir fielmente as palavras do estudo de PINHEIRO (1994). Além disso, na presente pesquisa, os dados considerados relevantes estão relacionados à variável "freqüência de ocorrência" e não à variável "regularidade da palavra” (cf. Resultados e Discussão). 
classificadas como regular, regra e irregular (do mesmo modo que as palavras das quais derivaram). Por exemplo, a palavra inventada dasa, derivada de casa, foi classificada como regra. Da mesma forma, nezena, derivada de dezena, recebe a classificação de irregular.

Na leitura, a pronúncia correta de uma palavra inventada deve respeitar o seguinte: (1) o condicionamento contextual (ex.: dasa lida como /'daza/, mas não como /'dassa/); (2) a pronúncia da vogal, como indicada pelo acento (ex.: dalé lida como /dalé/ e não como /dalê/ ou como /'dale/).

$\mathrm{O}$ total de palavras que compõem a tarefa está dividido em:

- $\quad$ seis palavras regulares de alta freqüência: festa, papai, chuva, café, folhas, palavra;

- seis palavras regulares de baixa freqüência: vila, cabras, batalha, jipe, pesca, moeda;

- seis palavras regulares inventadas: vesta, bavai, chuda, dalé, dolhas, calafra;

- $\quad$ seis palavras-regra de alta freqüência: casasa, galinha, pássaro disse, também, redação;

- $\quad$ seis palavras-regra de baixa freqüência: nora, empada, usam, quietos, florido, marreca;

- seis palavras-regra inventadas: daşa, gavinha, jássaco, sissi, dampém, mepação;

- $\quad$ seis palavras irregulares de alta freqüência: hoje, feliz, amanhã, onça, mamãe, dezena;

- seis palavras irregulares de baixa freqüência: boxe, açude, hino, luzes, xerife, descida;

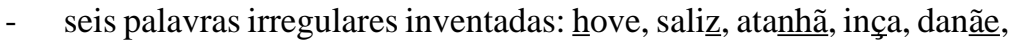
nezena.

\section{Resultados}

\section{Medidas de controle}

A tabela 2 mostra a média com o respectivo desvio padrão dos escores obtidos na tarefa de memória verbal, bem como a média e o desvio padrão dos escores $\mathrm{Z}$ no teste das Matrizes Progressivas de Raven. 
TABELA 2 - MÉDIA E DESVIO PADRÃO DOS ESCORES OBTIDOS NA TAREFA DE MEMÓRIA VERBALE NO TESTE DAS MATRIZES PROGRESSIVAS

\begin{tabular}{|c|c|c|c|c|c|c|}
\hline \multirow[b]{2}{*}{ Grupo } & \multirow[b]{2}{*}{$\mathrm{N}$} & \multirow[b]{2}{*}{$\begin{array}{l}\text { Idade } \\
\text { (média) }\end{array}$} & \multicolumn{2}{|c|}{$\begin{array}{l}\text { Memória } \\
\text { Verbal } \\
\text { (12 sentenças) }\end{array}$} & \multicolumn{2}{|c|}{$\begin{array}{c}\text { Raven } \\
\text { (escores Z) }\end{array}$} \\
\hline & & & M. & D. P. & M. & D. P. \\
\hline 1 & 20 & 11a. $6 \mathrm{~m}$. & 9,00 & 1,12 & 0,52 & 0,40 \\
\hline 2 & 20 & 7a. $1 \mathrm{~m}$. & 8,10 & 1,17 & 0,63 & 0,52 \\
\hline 3 & 20 & 11a. $5 \mathrm{~m}$. & 9,00 & 1,34 & 0,54 & 0,40 \\
\hline Total & 60 & 10a. & 8,70 & 1,27 & 0,56 & 0,44 \\
\hline
\end{tabular}

Como se pode observar na tabela 2, os escores em memória verbal estão mais relacionados à idade do que ao nível de leitura.

A comparação das médias utilizando a ANOVA (one-way) mostrou que a diferença entre os grupos é significativa $[\mathrm{F}(2,57)=3,67 ; \mathrm{p}<0,03]$. Entretanto, a comparação dos grupos em pares, usando o teste post hoc de Scheffé, mostrou que não existe nenhum par em que os grupos sejam significativamente diferentes. Assim, infere-se que qualquer diferença de desempenho entre os grupos na tarefa de leitura não pode ser atribuída à memória verbal.

Em relação ao teste das Matrizes Progressivas, os resultados demonstram que o grupo 2 tem uma média ligeiramente superior aos outros dois grupos. No entanto, a comparação das médias utilizando-se a ANOVA (oneway) mostrou que essa diferença não é significativa $[\mathrm{F}(2,57)=0,36 ; \mathrm{p}>$ $0,70]$. Portanto, os resultados indicam que quaisquer diferenças que venham a ser encontradas na tarefa de leitura não poderão ser atribuídas às habilidades medidas pelo teste de RAVEN.

\section{Tarefa de leitura de palavras isoladas}

Na tabela 3 são apresentados os escores obtidos pelos sujeitos de cada um dos três grupos, na tarefa de leitura de palavras isoladas. 
TABELA 3 - MÉDIA E DESVIO PADRÃO DOS ESCORES DE LEITURA (POR CONDIÇÃO E NO TOTAL)

\begin{tabular}{|c|c|c|c|c|c|c|c|c|c|}
\hline \multirow{3}{*}{ Grupos } & \multirow{3}{*}{$\mathrm{N}$} & \multicolumn{8}{|c|}{ Leitura de palavras } \\
\hline & & \multicolumn{2}{|c|}{$\begin{array}{l}\text { Alta freqüência } \\
\text { (18 palavras) }\end{array}$} & \multicolumn{2}{|c|}{$\begin{array}{c}\text { Baixa freqüência } \\
\text { (18 palavras) }\end{array}$} & \multicolumn{2}{|c|}{$\begin{array}{l}\text { Inventadas } \\
\text { (18 palavras) }\end{array}$} & \multicolumn{2}{|c|}{$\begin{array}{c}\text { Total } \\
\text { (54 palavras) }\end{array}$} \\
\hline & & M. & D. P. & M. & D. P. & M. & D. P. & & D. P. \\
\hline Grupo 1 & 20 & 15,55 & 2,80 & 13,05 & 2,37 & 9,70 & 2,68 & 38,30 & 6,73 \\
\hline Grupo 2 & 20 & 15,25 & 2,81 & 13,35 & 2,78 & 12,10 & 3,74 & 40,70 & 8,38 \\
\hline Grupo 3 & 20 & 18,00 & - & 17,15 & 0,81 & 15,35 & 1,60 & 50,50 & 1,85 \\
\hline Total & 60 & 16,27 & 2,57 & 14,52 & 2,84 & 12,38 & 3,62 & 43,17 & 8,16 \\
\hline
\end{tabular}

A tabela 3 mostra que, como se esperava, os sujeitos do grupo 3 tiveram um melhor desempenho que os sujeitos dos outros grupos, em todas as condições. Além disso, como pode ser observado, independentemente do grupo, os sujeitos tiveram melhor desempenho na leitura de palavras de alta freqüência do que na leitura de palavras de baixa frequiência e melhor desempenho nesta do que na leitura de palavras inventadas. Para confirmar esses resultados, foi efetuada uma Análise de Variância para medidas repetidas, tendo como fator intra-sujeitos o efeito freqüência (alta freqüência, baixa freqüência e palavras inventadas) e, como fator inter-sujeitos, os grupos. Essa análise mostrou uma diferença significativa no desempenho dos três grupos $[\mathrm{F}(2,57)=21,08 ; \mathrm{p}<$ 0,001]. Com a utilização do teste post hoc de Tukey, confirma-se que as diferenças entre os grupos se devem ao desempenho significativamente melhor do grupo 3 em relação aos outros dois grupos; não existe diferença significativa entre os grupos 1 e 2 . A análise mostrou também um efeito significativo do fator freqüência $[\mathrm{F}(2,114)=98,68 ; \mathrm{p}<0,001]$. A análise das diferenças entre as médias, por meio do teste post hoc de Tukey, confirma que o efeito significativo de freqüência se deve a um desempenho significativamente melhor na leitura de palavras de alta frequiência do que na leitura de palavras de baixa freqüência, e nestas, melhor do que na leitura de palavras inventadas.

Foi observado, também, um efeito significativo de interação Grupo X Frequiência $[\mathrm{F}(4,114)=7,03 ; \mathrm{p}<0,001]$. Esse efeito se deve ao fato de $\mathrm{o}$ grupo 1 demonstrar um pior desempenho que o grupo 2 apenas na leitura de "palavras inventadas". Isso pode ser confirmado pelo percentual médio de acertos dos diferentes grupos em relação à leitura das palavras reais e inventadas (cf. tabela 4). 
TABELA 4 - MÉDIA DOS PERCENTUAIS DE ACERTOS NA LEITURA DAS PALAVRAS REAIS E INVENTADAS, POR GRUPO

\begin{tabular}{cccccccc}
\hline & & \multicolumn{5}{c}{ Percentual de acertos na leitura } \\
\cline { 3 - 8 } Grupo & & \multicolumn{2}{c}{$\begin{array}{c}\text { Palavras reais } \\
\text { (36 palavras) }\end{array}$} & \multicolumn{2}{c}{ Palavras inventadas } & \multicolumn{2}{c}{ Total } \\
& N & M. & D. P. & M. & D. P. & M. & D. P. \\
\hline 1 & 20 & 79,44 & 13,20 & 53,89 & 14,87 & 70,93 & 12,46 \\
2 & 20 & 79,44 & 14,43 & 67,22 & 20,78 & 75,37 & 15,52 \\
3 & 20 & 97,64 & 2,26 & 85,28 & 8,88 & 93,52 & 3,43 \\
\hline Total & 60 & 85,51 & 14,13 & 68,80 & 20,10 & 79,94 & 15,11 \\
\hline
\end{tabular}

Destaca-se que não existe diferença na leitura de palavras reais entre os grupos 1 e 2 (o que era de se esperar, uma vez que eles foram emparelhados pelo nível de leitura).

Verifica-se, em todos os grupos, uma preferência pelo procedimento visual de leitura, uma vez que os três grupos preenchem as condições que o caracteriza: (1) melhor desempenho na leitura de palavras de alta frequiência do que na leitura de palavras de baixa freqüência; (2) melhor desempenho na leitura de palavras reais do que na leitura de palavras inventadas.

Entretanto, embora os três grupos mostrem um desempenho superior na leitura de palavras reais do que na leitura de palavras inventadas, esta diferença é maior no grupo 1 do que nos outros grupos. Portanto, os dados recolhidos apontam que, considerando-se os três grupos, os sujeitos do grupo 1 apresentam a menor capacidade em utilizar a mediação fonológica (relação letrasom) na leitura. Ainda assim, a capacidade de decodificação dos referidos sujeitos ultrapassa $50 \%$ das palavras inventadas, o que demonstra que, embora eles apresentem dificuldade na utilização da rota sublexical (fonológica) de leitura, não deixam de utilizá-la.

\section{Discussão}

As análises efetuadas em relação à tarefa de leitura procuraram averiguar como o desempenho dos três grupos avaliados era afetado pela freqüên- 
cia de ocorrência das palavras apresentadas para a leitura. Verificou-se, desse modo, qual era o procedimento de leitura utilizado preferencialmente pelos sujeitos dos diferentes grupos. A média dos escores obtidos, por grupo, revelou que, no geral, todos os sujeitos tiveram melhor desempenho na leitura das palavras de alta frequiência e pior desempenho na leitura de palavras inventadas. Esses resultados podem ser interpretados como indicativos da utilização preferencial de um procedimento visual (não-analítico) por parte de todos os grupos. Portanto, não se verifica uma diferença entre os procedimentos utilizados pelos sujeitos com e sem dificuldades de aprendizagem, mesmo quando se considera o grupo 3, no qual os sujeitos apresentam um nível de leitura e de escrita mais avançado do que o dos sujeitos dos outros grupos.

Entretanto, verifica-se que o grupo 1 distingue-se dos demais em relação à capacidade de utilizar a mediação fonológica na leitura, ou seja, os sujeitos do grupo 1 manifestaram menor facilidade do que os outros sujeitos na utilização da rota sublexical (fonológica) de leitura.

Contudo, apesar de os sujeitos do grupo 1 terem demonstrado uma menor capacidade do que os outros sujeitos em efetuar uma mediação fonológica (utilizar a rota sublexical) na leitura, é importante destacar que esses resultados são diferentes dos habitualmente encontrados na literatura de língua inglesa, que apontam uma grande diferença entre a capacidade dos disléxicos na leitura de palavras e não-palavras. Por exemplo, no estudo de BADDELEY et al. (1982), os disléxicos apresentaram na leitura de palavras reais uma média de erros de 6,3\% contra $41,6 \%$ de erros na leitura de palavras inventadas; portanto, uma relação maior do que 1 para 6 . No presente estudo, os sujeitos do grupo 1 apresentaram na leitura de palavras reais de alta freqüência (aquelas que supostamente teriam sido lidas pela rota lexical) uma média de erros de $13,61 \%$ contra $46,11 \%$ na leitura de palavras inventadas, numa relação equivalente a 1 para 3 .

A partir dessas considerações, conclui-se que os sujeitos falantes do Português que apresentam dificuldades de aprendizagem utilizam os mesmos procedimentos de leitura dos leitores "normais". Ou seja, todas as crianças usam uma combinação dessas duas estratégias de leitura (procedimento lexical e sublexical), dependendo das palavras que o contexto oferece.

Comparando os resultados desta pesquisa com os de outras, as quais estudaram sujeitos falantes de outras línguas que não o Português, houve indícios de que os problemas dos sujeitos com dificuldades de leitura manifestam-se, se não de formas distintas, com diferentes intensidades. Portanto, aponta-se a necessidade de realizar novos estudos, sobretudo estudos longitudinais, para identificar como essas dificuldades vão se alterando com o de- 
senvolvimento da criança e com o aumento da sua escolaridade. Nesse sentido, sugere-se que as explicações do padrão de leitura dos sujeitos com dificuldades devem ser buscadas nos modelos e descrições do próprio processo de aquisição e desenvolvimento da leitura e da escrita. Acredita-se que esse tipo de estudo poderá esclarecer melhor como e quando são desenvolvidas as múltiplas habilidades cognitivas necessárias para o domínio pleno da leitura.

\section{REFERÊNCIAS}

ALVARENGA, D. Leitura e escrita: dois processos distintos. Educação em Revista, Belo Horizonte, v. 7, p. 27-31, 1988.

BADDELEY, A. D. et al. Developmental and acquired dyslexia: a comparison. Cognition, Lausanne, v. 11, p. 185-199, 1982.

BADDELEY, A. D.; LOGIE, R. H.; ELLIS, N. C. Characteristics of developmental dyslexia. Cognition, Amsterdam, v. 29, p. 197-228, 1988.

BARRERA, S. D. Linguagem oral e alfabetização: um estudo sobre variação lingüística e consciência metalingüística em crianças da $1^{a}$ série do ensino fundamental. São Paulo, 2000. 225 p. Tese (Doutorado em Psicologia) - Instituto de Psicologia da Universidade de São Paulo.

BEHRMANN, M.; BUB, D. Surface dyslexia and dysgraphia: dual routes, single lexicon. Cognitive Neuropsychology, v. 9, n. 3, p. 209-251, 1992.

BODER, E. Developmental dyslexia: a diagnostic approach based on three atypical reading-spelling patterns. Developmental Medicine and Child Neurology, London, v. 15, p. 663-687, 1973.

BRYANT, P. E.; BRADLEY, L. Problemas de aprendizagem de leitura. Porto Alegre: Artes Médicas, 1987.

BRYANT, P. E.; IMPEY, L. The similarities between normal readers and developmental and acquired dyslexics. Cognition, v. 24, p. 121-137, 1986.

BRYANT, P. E.; NUNES, T.; BINDMAN, M. Backward readers' awareness of language: strengths and weaknesses. European Journal of Psychology of Education, v. 12 , n. 4 , p. $357-372,1997$.

CAPOVILLA, A. G. S. Leitura, escrita e consciência fonológica: desenvolvimento intercorrelações e intervenções. São Paulo, 1999, 262 p. Tese (Doutorado em Psicologia) - Instituto de Psicologia da Universidade de São Paulo. 
CASTLES, A.; COLTHEART, M. Varieties of developmental dyslexia. Cognition, Amsterdam, v. 47, p. 149-180, 1993.

COLTHEART, M. Varieties of developmental dyslexia: a comment on Bryant and Impey. Cognition, Amsterdam, v. 27, n. 1, p. 97-101, 1987.

COLTHEART, M. et al. Surface dyslexia. Quarterly Journal of Experimental Psychology, v. 35A, p. 469-495, 1983.

CURITIBA. Prefeitura Municipal de Curitiba. Secretaria Municipal de Educação. Proposta de Educação Especial na Secretaria Municipal de Educação. Curitiba, 1992.

ELLIS, A. W. Leitura, escrita e dislexia: uma abordagem cognitiva. 2 ed. Porto Alegre: Artes Médicas, 1995.

FRITH, U. Beneath the surface of developmental dyslexia. In: PATTERSON, K.; COLTHEART, M.; MARSHALL, J. C. (Eds.). Surface dyslexia: Neuropsychological and cognitive studies of phonological reading. London: Lawrence Erlbaum Associates, 1984, p. 301-330.

GUIMARÃES, S. R. K. Dificuldades na aquisição e aperfeiçoamento da leitura e da escrita: o papel da consciência fonológica e da consciência sintática. São Paulo, 2001, 269 p. Tese (Doutorado em Psicologia) - Instituto de Psicologia da Universidade de São Paulo.

GUTHRIE, J. T. Reading comprehension and syntactic responses in good and poor readers. Journal of Educational Psychology, v. 65, n. 3, p. 294-299, 1973.

MARSHALL, J. C.; NEWCOMBE, F. Patterns of paralexia: a psycholinguistic approach. Journal of Psycholinguist Research, v. 2, n. 3, p. 175-199, 1973.

MORAIS, J. A arte de ler. São Paulo: Unesp, 1996.

PARTZ, M. P. A avaliação da leitura em neuropsicologia. In: GRÉGOIRE, J.; PIÉRART, B. Avaliação dos problemas de leitura: os novos modelos teóricos e suas implicações diagnósticas. Porto Alegre: Artes Médicas, 1997. p. 53-64.

PINHEIRO, A. M. V. Leitura e escrita: uma abordagem cognitiva. Campinas: Editorial Psy II, 1994.

RACK, J. P.; SNOWLING, M. J.; OLSON, R. K. The nonword reading deficit in developmental dyslexia: a review. Reading Research Quarterly, Newark, v. 27, n. 1, p. 29-53, 1992.

RAVEN, J. C. Manual teste das matrizes progressivas: escala geral. Tradução: Francisco Campos. Rio de Janeiro: CEPA, 1999.

REGO, L. L. B. Diferenças individuais na aprendizagem inicial da leitura: papel desempenhado por fatores metalingüísticos. Psicologia: Teoria e Pesquisa, Brasília, v. 11, n. 1, p. 51-60, 1995. 
GUIMARÃES, S. R. K. Dislexias adquiridas como referência...

STEIN, L. M. TDE: teste de desempenho escolar: manual para aplicação e interpretação. São Paulo: Casa do Psicólogo, 1994.

TEMPLE, C. M.; MARSHALL, J. C. A case study of developmental phonological dyslexia. British Journal of Psychology, v. 74, p. 517-533, 1983.

Texto recebido em 20 ago. 2003 Texto aprovado em 03 abr. 2004 